Trascender, Contabilidad y Gestión. Vol. 6, Núm. 18 (septiembre - diciembre del 2021).

Universidad de Sonora. Departamento de Contabilidad. México.

ISSN: 2448-6388. Reserva de Derechos 04-2015-04172070800-203.

\title{
La gestión del conocimiento, pasado, presente y futuro. Una revisión de la literatura
}

\author{
The management of knowledge, past, present and future. A literature reviews \\ Laura Margarita Villasana Arreguín ${ }^{1}$; Patricia Hernández García ${ }^{2}$; Élfego Ramírez Flores ${ }^{3}$
}

Recibido: 16 de febrero de 2021.

Aceptado: 6 de mayo de 2021.

DOI: https://doi.org/10.36791/tcg.v0i18.128

JEL: D83. Información y conocimiento.

M10. Administración de empresas, generalidades.

D20. Producción y organizaciones, generalidades.

\section{Resumen}

Desde sus inicios, la gestión del conocimiento siempre ha sido el campo más interesante para académicos y profesionales, y se observa como una esperanza de mejora dentro de la organización. Uno de los retos de la gestión del conocimiento es gestionarlo desde el proceso de creación, intercambio, combinación y aplicación del conocimiento para aclarar y potenciar el proceso organizacional. Esta investigación tiene como objetivo proporcionar una visión teórica del concepto de gestión del conocimiento, sus orígenes, modelos, aplicaciones, estatus quo y el futuro para resaltar su importancia e impacto en la organización. Al revisar los artículos científicos, el análisis de la literatura se utiliza como método de investigación. Como resultado, se obtuvo el último nivel técnico de gestión del conocimiento terminológico, su modelo de futuro y una síntesis aproximada.

Palabras clave: conocimiento, gestión, modelos, organizaciones.

\footnotetext{
${ }^{1}$ Laura Margarita Villasana Arreguín. Doctora en Administración. Investigador independiente del Doctorado en la Universidad Autónoma de San Luis Potosí, Facultad de Contaduría y Administración. Correo: a6552@alumnos.uaslp.mx. ORCID: https://orcid.org/0000-0002-4592-7550

2 Patricia Hernández García. Doctora en Administración. Profesor de Tiempo Completo, Facultad de Contaduría y Administración, Universidad Autónoma de San Luis Potosí. Correo: patricia.hernandez@uaslp.mx. ORCID: https://orcid.org/0000-0002-6214-8268

3 Élfego Ramírez Flores. Maestro en Administración. Profesor de Tiempo Completo, Facultad de Contaduría y Administración, Universidad Autónoma de San Luis Potosí. Correo: elfego@uaslp.mx. ORCID: https://orcid.org/0000-0002-9483-6997
}

Villasana Arreguín, L. M., Hernández García, P., y Ramírez Flores, E.
Vol. 6, núm. 18 / septiembre - diciembre del 2021 DOI: https://doi.org/10.36791/tcg.v0i18.128

Pp. 53-78 


\section{Abstract}

Since the beginning knowledge management has always been the most interesting field for academics and professionals, and it is seen as a hope for improvement within the organization. One of the challenges of knowledge management is to manage it from the process of creation, exchange, combination, and application of knowledge to clarify and enhance the organizational process. This research aims to provide a theoretical view of the concept of knowledge management, its origins, models, applications, status quo, and the future to highlight its importance and impact on the organization. When reviewing scientific articles, literature analysis is used as a research method. As a result, the latest technical level of terminology knowledge management, its future model and an approximate synthesis were obtained.

Keywords: knowledge, management, models, organizations.

\section{Introducción}

Algunas personas creen que la gestión del conocimiento es indispensable debido a la creciente globalización, la velocidad de la información y el conocimiento, la dinámica de la innovación de productos y procesos y los cambios ambientales provocados por la competencia del mercado (Picot, 1998, citado por Greiner et al., 2007). La gestión del conocimiento se dedica a ayudar a las empresas a ser más rápidas, más eficientes o innovadoras que sus competidores. Además, la gestión del conocimiento se ocupa de la interacción entre la organización y el entorno, así como la capacidad de respuesta y acciones de la organización (Macharzina, 1999; Greiner et al., 2007). Entre los elementos básicos de una organización, el conocimiento es considerado un recurso importante que ayuda a crear valor (Barney, 1991; Greiner et al., 2007).

Las organizaciones que son conscientes de sus diferentes recursos de conocimiento tienen claves únicas y valiosas que son difíciles de imitar y que pueden utilizarse para lograr ventajas competitivas sostenibles (Alavi y Leidner, 2001; Greiner et al., 2007). Este también es un medio para adquirir, mantener y hacer un uso completo del conocimiento de los recursos, lo que puede aumentar el nivel de éxito de la organización. Por lo tanto, la gestión del conocimiento incluye todas las actividades que utilizan el conocimiento para alcanzar las metas organizacionales para enfrentar los desafíos ambientales y mantener su competitividad en el mercado (Greiner et al., 2007).

Stankosky (2005, citado en Holtshouse, 2010) define la gestión del conocimiento como el uso de los activos de conocimiento más relevantes que permite mejorar el desempeño, enfocado en la eficiencia, la eficacia y la innovación. La gestión del conocimiento es estratégicamente importante y esencial para el éxito de la organización (Edmonson, 2010). La gestión del conocimiento es el tema principal de las organizaciones, su foco está en sus esfuerzos para promover la creación, intercambio, combinación y aplicación del conocimiento para lograr una ventaja competitiva, pero aún faltan métodos de seguimiento para determinar el enfoque de la empresa. Abordar el 
conocimiento tácito, la teoría de recursos y capacidades y la creación de conocimiento organizacional son elementos valiosos para que las empresas maximicen sus capacidades (Tenorio, Popadiuk y Santos, 2019).

Algunas de las preguntas que esta investigación necesita responder son: ¿Qué es la gestión del conocimiento? ¿Cuál es la teoría en la que se basa la gestión del conocimiento? ¿Cuáles son los modelos de gestión del conocimiento? ¿Cómo aplicar la gestión del conocimiento? ¿Cuáles son los beneficios de la gestión del conocimiento? ¿Cuáles son sus expectativas para la gestión del conocimiento? Por tanto, el propósito de esta investigación es realizar una revisión teórica del concepto de gestión del conocimiento, su origen, modelo, aplicación, situación actual y desarrollo futuro, con el fin de resaltar su importancia e impacto en la organización.

La importancia de esta investigación radica en mostrar las diferentes definiciones de gestión del conocimiento y sus similitudes. Estas definiciones están relacionadas con la creación, intercambio, combinación y aplicación del conocimiento, las cuales están implícitas en la teoría de recursos y capacidades (Penrose, 1959; y Barney, 1991); y la teoría de la creación de conocimiento organizacional propuesta por Nonaka y Takeuchi (Nonaka y Takeuchi, 1995). Enfatiza la discusión actual orientada a comprender las peculiaridades de estas dos teorías de creación y gestión del conocimiento, a fin de brindar alguna ayuda desde el punto de vista académico, y buscar identificar modelos de gestión que ayuden a difundir el conocimiento en la organización. La investigación describe el concepto de gestión del conocimiento, las principales teorías relacionadas, modelos, aplicaciones y algunos métodos para realizar su futuro.

\section{La integración de la gestión del conocimiento}

\section{El conocimiento}

En principio, el conocimiento como cultura se difunde y adquiere de tres maneras, como cualquier proceso de comunicación: razón / lenguaje; experiencia / acción; y perceptual / simbólica. Una de las características de los excelentes trabajadores del conocimiento es que combinan bien estos tres aspectos en el aprendizaje y la comunicación (Abellon y Rojo, 2004). A medida que el conocimiento adquiere cada vez más importancia como un nuevo factor de producción, el conocimiento se ha convertido en un elemento básico del desarrollo económico y social, porque el conocimiento se produce a partir de una combinación de información, experiencia, valor y normativas internas (Rodríguez, 2006; Davenport y Prusak, 2001, citado en Romero, 2016).

Como describen Abeson y Taku (2009), el conocimiento utilizado como recurso competitivo tiene un efecto positivo, e implica que la importancia del conocimiento tácito y explícito para la toma de decisiones proporciona un marco para que las pequeñas empresas adquieran conocimiento de la compañía. El conocimiento tácito se genera a través de la experiencia, que es un conocimiento subjetivo natural, y a menudo participa simultáneamente con la experiencia (Dooley et al., 1998; Abeson y Taku, 2009). Según Polanyi (1966, por Abeson y Taku, 2009) y Nonaka y Takeuchi (1995), el conocimiento tácito es
Villasana Arreguín, L. M., Hernández García, P., y Ramírez Flores, E. 
abstracto y solo puede ser comunicado mediante la participación. Berman et al. (2002; Citado por Abeson y Taku, 2009) y Grant (1996; citado por Abeson y Taku, 2009) definen el conocimiento tácito como conocimiento basado en la experiencia, que es difícil de codificar, copiar y difundir.

El conocimiento definido es objetivo por naturaleza (Abeson y Taku, 2009). Según Polanyi (1966; citado en Abeson y Taku, 2009), y Nonaka y Takeuchi (1995) también son altamente codificables y difundidos en lenguajes formales $\mathrm{y}$ sistemáticos. Berman et al. (2002); Grant (1996) como se citaron en Abeson y Taku (2009) definen el conocimiento explícito como el conocimiento basado en hechos y teorías, el cual se puede codificar, copiar y difundir más fácilmente a otros. En resumen, McAulay et al. (1997, como se citó en Abeson y Taku, 2009), el conocimiento claro puede expresarse claramente y comunicarse abiertamente y quedar escrito en documentos.

\section{La gestión del conocimiento}

Desde la definición y perspectiva del conocimiento considerado como un activo organizacional, la gestión del conocimiento es un método de gestión o disciplina emergente que busca utilizar el conocimiento generado de manera estructurada y sistemática para alcanzar metas y optimizar la toma de decisiones. Sin embargo, la gestión del conocimiento es un concepto en construcción, con diversas interpretaciones, y sigue basándose en trabajos de investigación (Avendaño y Flores, 2016). Borroto (2007); Belinza, Guerrero, Colonia y Ramírez (2011); mencionaron que el conocimiento gerencial significa la creación, desarrollo, difusión y utilización del conocimiento gerencial para obtener y asegurar su viabilidad y supervivencia, que se conceptualiza como conocimiento clave.

Existen múltiples definiciones sobre la gestión del conocimiento, entre las cuales los autores más famosos son Bueno (1998), Davenport y Prusak (1998) y Sveiby (2018). En la tabla 1 se muestra algunas definiciones de gestión del conocimiento en la literatura.

\section{Tabla 1}

Definiciones de gestión del conocimiento

\begin{tabular}{|l|l|}
\hline \multicolumn{1}{|c|}{ Autor } & \multicolumn{1}{c|}{ Definición } \\
\hline $\begin{array}{l}\text { Davenport } \\
\text { Prusak (1998) }\end{array}$ & $\begin{array}{l}\text { La gestión del conocimiento se refiere a las acciones que realiza la organización con el fin de } \\
\text { obtener el máximo valor del conocimiento disponible. }\end{array}$ \\
\hline Ives et al., (1997) & $\begin{array}{l}\text { En el último sentido, la gestión del conocimiento generalmente puede verse como el trabajo, } \\
\text { las personas, el tiempo, y la forma que la organización necesitan el conocimiento. }\end{array}$ \\
\hline
\end{tabular}

Villasana Arreguín, L. M., Hernández García, P., y Ramírez Flores, E. 


\section{Tabla 1}

Continuación...

\begin{tabular}{|c|c|}
\hline Autor & Definición \\
\hline Rosenberg (2006) & $\begin{array}{l}\text { La gestión del conocimiento es el establecimiento y archivo de información, experiencia y } \\
\text { conocimiento valiosos dentro y entre comunidades de personas y organizaciones con intereses } \\
\text { y necesidades similares, y su propósito es crear una ventaja competitiva. }\end{array}$ \\
\hline Wiig (1997) & $\begin{array}{l}\text { La gestión del conocimiento se refiere a la creación, renovación y aplicación de un } \\
\text { conocimiento claro y consciente que comprende, presta atención y gestiona el sistema. }\end{array}$ \\
\hline $\begin{array}{l}\text { Bair y O’Connor } \\
\text { (1998) }\end{array}$ & $\begin{array}{l}\text { La tecnología de gestión del conocimiento es la integración de una serie de productos de } \\
\text { software, incluida la recuperación de información, el trabajo en grupo y la gestión de } \\
\text { documentos. }\end{array}$ \\
\hline $\begin{array}{l}\text { Coulson-Thomas } \\
\text { (1997) }\end{array}$ & $\begin{array}{l}\text { La gestión del conocimiento se ha convertido en un problema de acceso a formas específicas de } \\
\text { conocimiento, especialmente datos e información, que se almacenan electrónicamente o en } \\
\text { ubicaciones definidas. }\end{array}$ \\
\hline Bair (1997) & $\begin{array}{l}\text { La gestión del conocimiento tiene como objetivo capturar el conocimiento que los empleados } \\
\text { realmente necesitan en un repositorio central y filtrar el conocimiento restante. }\end{array}$ \\
\hline $\begin{array}{l}\text { Fenn (1996 como } \\
\text { se citó en Morey, } \\
\text { 2001) }\end{array}$ & $\begin{array}{l}\text { La gestión del conocimiento promueve un método integrado para identificar, capturar, } \\
\text { recuperar y evaluar los activos de información de la empresa. Estos activos de información } \\
\text { pueden incluir bases de datos, documentos, políticas, procesos y experiencias predeterminadas } \\
\text { no capturadas y experiencias almacenadas en la mente de las personas. }\end{array}$ \\
\hline $\begin{array}{l}\text { Saint-Onge } \\
(2000)\end{array}$ & $\begin{array}{l}\text { Es la capacidad de desarrollar, mantener, influir y actualizar activos intangibles denominados } \\
\text { capital de conocimiento o capital de conocimiento. }\end{array}$ \\
\hline Sveiby (1998). & $\begin{array}{l}\text { El arte de utilizar activos intangibles para crear valor en una organización. El arte de crear } \\
\text { valor mediante la mejora de los activos intangibles y el tratamiento de las organizaciones como } \\
\text { conocimiento omnipresente y flujo de conocimiento }\end{array}$ \\
\hline
\end{tabular}

Villasana Arreguín, L. M., Hernández García, P., y Ramírez Flores, E.
Vol. 6, núm. 18 / septiembre - diciembre del 2021 DOI: https://doi.org/10.36791/tcg.v0i18.128

Pp. 53-78 


\section{Tabla 1}

Continuación...

\begin{tabular}{|l|l|}
\hline \multicolumn{1}{|c|}{ Autor } & \multicolumn{1}{|c|}{ Definición } \\
\hline Borroto (2007). & $\begin{array}{l}\text { La gestión del conocimiento puede describirse como el proceso sistemático de organizar a los } \\
\text { participantes para que detecten, seleccionen, organicen, filtren, presenten y utilicen información } \\
\text { con el fin de desarrollar recursos de conocimiento basados en el propio capital intelectual de la } \\
\text { organización, con el objetivo de mejorar las habilidades organizativas y crear valor. }\end{array}$ \\
\hline Bueno (1998) & $\begin{array}{l}\text { La gestión del conocimiento de la empresa se compone esencialmente de tres dimensiones } \\
\text { conceptuales: un conjunto de conocimiento explícito y tácito capturado y creado, tecnología TIC } \\
\text { que promueve el proceso, y procesos estratégicos organizacionales de generación de } \\
\text { conocimiento dinámico. }\end{array}$ \\
\hline $\begin{array}{l}\text { Logan } \\
\text { Ortiz de Urbina, } \\
\text { (2000). }\end{array}$ & $\begin{array}{l}\text { Es el uso de información estratégica para lograr los objetivos comerciales y organizar } \\
\text { actividades para crear un entorno social y una infraestructura para que se pueda acceder, } \\
\text { compartir y crear el conocimiento. } \\
\text { conocimiento para identificar y utilizar los activos intangibles existentes y generar nuevos } \\
\text { Fainholc, (2006) }\end{array}$ \\
\hline $\begin{array}{l}\text { Minakata } \\
\text { (2009). }\end{array}$ & $\begin{array}{l}\text { Leste el momento en que los empleados expresan claramente su conocimiento a la } \\
\text { la organización. } \\
\text { través de la infraestructura y los intermediarios de comunicación, de modo que el conocimiento } \\
\text { generado sea significativo para la empresa. }\end{array}$ \\
\hline
\end{tabular}

Villasana Arreguín, L. M., Hernández García, P., y Ramírez Flores, E. 


\section{Tabla 1}

Continuación...

\begin{tabular}{|c|c|}
\hline Autor & Definición \\
\hline $\begin{array}{l}\text { Mijangos Noh, } \\
\text { Cabrera y Sugey } \\
(2012)\end{array}$ & $\begin{array}{l}\text { La gestión del conocimiento es un proceso estratégico, guiado y operado por la disciplina y las } \\
\text { habilidades, que promueve ventajosamente la realización de los objetivos establecidos. Este } \\
\text { proceso se utiliza para poner en práctica el conocimiento tácito y el conocimiento explícito y } \\
\text { maximizar el valor de la organización. La gestión del conocimiento ayuda a la toma de } \\
\text { decisiones, aumentando así la viabilidad y durabilidad de la organización, y generando ventajas } \\
\text { competitivas. Para lograrlo, la información y el conocimiento deben estar disponibles y } \\
\text { accesibles para que los miembros de la organización puedan absorberlos y convertirlos en } \\
\text { experiencia. }\end{array}$ \\
\hline Alvarado (2005) & $\begin{array}{l}\text { La gestión del conocimiento tiene una perspectiva táctica y operativa, es más detallada y se } \\
\text { centra en la promoción y gestión de las actividades relacionadas con el conocimiento, como la } \\
\text { creación, adquisición, transformación y uso del conocimiento. Su función es planificar, } \\
\text { implementar, operar, dirigir y controlar todas las actividades relacionadas con el conocimiento y } \\
\text { los procedimientos, que son necesarios para una gestión eficaz del capital intelectual. }\end{array}$ \\
\hline Borroto (2007). & $\begin{array}{l}\text { La gestión de la información sustentada en las nuevas tecnologías de la organización y una } \\
\text { buena cultura organizacional, y el proceso sistemático de mejora de sus habilidades a través del } \\
\text { capital intelectual de la organización, generando así una ventaja competitiva. El conocimiento } \\
\text { de gestión es la gestión de todos los activos intangibles que agregan valor a la organización en } \\
\text { términos de lograr habilidades o capacidades básicas únicas. Por lo tanto, este es un concepto } \\
\text { dinámico, es decir, de flujo. }\end{array}$ \\
\hline $\begin{array}{l}\text { Calderón, (2017) } \\
\text { Fidalgo-Blanco } \\
\text { et al., (2014) }\end{array}$ & $\begin{array}{l}\text { La gestión del conocimiento en organizaciones como las instituciones de educación superior } \\
\text { significa la conversión de información en conocimiento, lo que conduce a un aumento en la } \\
\text { cantidad de producción de conocimiento organizacional. }\end{array}$ \\
\hline $\begin{array}{l}\text { Davenport } \\
(1999)\end{array}$ & $\begin{array}{l}\text { Es un proceso sistemático de búsqueda, organización, filtrado y presentación de información } \\
\text { para mejorar la comprensión de las personas sobre áreas específicas de interés. }\end{array}$ \\
\hline
\end{tabular}

Villasana Arreguín, L. M., Hernández García, P., y Ramírez Flores, E.
Vol. 6, núm. 18 / septiembre - diciembre del 2021 DOI: https://doi.org/10.36791/tcg.v0i18.128

Pp. $53-78$ 


\section{Tabla 1}

Continuación...

\begin{tabular}{|l|l|}
\hline \multicolumn{1}{|c|}{ Autor } & \multicolumn{1}{c|}{ Definición } \\
\hline $\begin{array}{l}\text { Malhotra } \\
(2008)\end{array}$ & $\begin{array}{l}\text { Encarna el proceso organizacional de buscar la combinación colaborativa de procesamiento de datos } \\
\text { e información a través de las capacidades de la tecnología de la información y la creatividad e } \\
\text { innovación humanas. }\end{array}$ \\
\hline
\end{tabular}

Fuente: Adaptada de Chatti, M. A. (2012).

En la tabla 1 se observan diferentes definiciones de gestión de conocimiento centrándose la mayoría de ellas en el proceso más que la descripción de esta. Para la presente investigación se considera la definición de Tom Davenport considerando que es el proceso de capturar, distribuir y utilizar de manera eficaz el conocimiento (Davenport, 1994).

\section{Teorías relacionadas con la gestión del conocimiento}

La teoría pionera de la gestión del conocimiento es la teoría de los recursos y las capacidades (Penrose, 1959; y Barney, 1991), centrándose en los recursos que las empresas pueden garantizar rendimientos normales sostenibles, incluidos los recursos estáticos, las capacidades dinámicas y el conocimiento (Kristandl y Bontis, 2007). Dabić, Lažnjak, Smallbone y Švarc (2018) enfatizaron que la teoría de recursos y capacidades llama la atención sobre la naturaleza de la coordinación interna de la empresa, la estructura y efectividad de la organización, y el rol de la gerencia y la asignación de poder y decisión. El último desarrollo teórico de una visión basada en el conocimiento o una visión de empresa basada en el capital del conocimiento (Reed et al., 2006) confirma que la capacidad de una empresa para innovar depende de su conocimiento y activos intelectuales (Martín de Castro, 2015; Subramaniam y Youndt, 2005; Ngah y Ibrahim, 2009; Secundo et al., 2017), que es una propuesta que es observable.

- Por otro lado, según la teoría de la creación de conocimiento organizacional, la organización de creación de conocimiento de Nonaka y Takeuchi (1995) tiene tres funciones principales: generar nuevo conocimiento, crear conocimiento, y transferir conocimiento. Difundirlo por toda la organización e integrar el conocimiento aprendido en las nuevas tecnologías. Según Nonaka y Takeuchi (1995), el conocimiento dentro de una organización puede residir a nivel individual o puede ser compartido por los miembros de la organización:

- El conocimiento personal es un tesoro de conocimiento "propiedad" de un individuo, que puede aplicarse de forma independiente a tipos específicos de tareas o problemas. Debido a que es con la persona, también se puede transferir, lo que puede generar problemas de retención de empleados y acumulación de conocimientos.
Villasana Arreguín, L. M., Hernández García, P., y Ramírez Flores, E. 
- El conocimiento colectivo se refiere a la forma en que los miembros de una organización distribuyen y comparten el conocimiento. Es el conocimiento acumulado de la organización, almacenado en las reglas, procedimientos, rutinas y especificaciones compartidas de la organización, que se utiliza para guiar las actividades de resolución de problemas y los modelos de interacción entre los miembros. Puede ser un conjunto de conocimiento almacenado como una base de datos fija, o puede ser un conocimiento de estado de flujo generado por interacción.

En términos concretos, el conocimiento es creado sólo por los individuos. Una organización no puede crear conocimiento sin individuos. La organización apoya la creatividad individual o provee el contexto para que los individuos generen conocimientos. Por lo tanto, la generación de conocimiento organizacional debe ser entendida como el proceso que amplifica organizacionalmente el conocimiento generado por los individuos y lo cristaliza como parte de la red de conocimientos de la organización. Por esto, la generación de conocimiento organizacional radica en el respaldo de la empresa, en torno a las potenciales fuentes de conocimiento: individuos, grupos, equipos, proyectos, áreas, departamentos, entre otras.

\section{Modelos de gestión del conocimiento}

Estos modelos reflejan la importancia del conocimiento en la organización, su complejidad, dinámica y la naturaleza estratégica de los activos intangibles. El conocimiento es considerado como el activo organizacional más valioso, el rol protagónico del capital humano y la integración de individuos, grupos y tecnología. Información y comunicación para lograr la mejor gestión del conocimiento en la organización. Estos modelos enfatizan que las organizaciones necesitan sistematizar y estructurar los procesos relacionados con el conocimiento y el flujo de información, y no dejar que se les presente ninguna oportunidad, porque cada uno de ellos es parte de la red de acción, debe tener vitalidad, el cual se muestra en la tabla 2 una comparación de modelos de gestión del conocimiento (Avendaño y Flores 2016) para mejorar la virtud del uso apropiado, integración o aplicación del conocimiento para lograr las metas organizacionales.
Villasana Arreguín, L. M., Hernández García, P., y Ramírez Flores, E.
Vol. 6, núm. 18 / septiembre - diciembre del 2021 DOI: https://doi.org/10.36791/tcg.v0i18.128

Pp. $53-78$ 
Tabla 2

Comparación de modelos de gestión del conocimiento

\begin{tabular}{|c|c|c|c|c|c|c|}
\hline Modelo & $\begin{array}{l}\text { Motor de la } \\
\text { GC }\end{array}$ & Uso de TIC & Contexto & Actores & $\begin{array}{c}\text { Tipo de } \\
\text { conocimiento }\end{array}$ & $\begin{array}{c}\text { Cultura } \\
\text { organizacional }\end{array}$ \\
\hline Wiig (1993) & TIC & $\begin{array}{l}\text { Uso de las TIC } \\
\text { en el proceso } \\
\text { de distribución } \\
\text { de } \\
\text { conocimiento }\end{array}$ & $\begin{array}{l}\text { Organizaciones } \\
\text { en general }\end{array}$ & $\begin{array}{l}\text { Miembros de } \\
\text { la } \\
\text { organización } \\
\text { Expertos }\end{array}$ & $\begin{array}{l}\text { Factual, } \\
\text { conceptual, } \\
\text { explicativo y } \\
\text { metodológico }\end{array}$ & $\begin{array}{l}\text { Abierta al } \\
\text { aprendizaje }\end{array}$ \\
\hline $\begin{array}{l}\text { Nonaka y } \\
\text { Takeuchi } \\
(1995)\end{array}$ & $\begin{array}{l}\text { Procesos } \\
\text { humanos }\end{array}$ & $\begin{array}{l}\text { Un medio, sin } \\
\text { inclusión } \\
\text { indispensable }\end{array}$ & Empresarial & $\begin{array}{l}\text { Individuos } \\
\text { Equipos de } \\
\text { trabajo }\end{array}$ & $\begin{array}{l}\text { Tácito } \\
\text { Explícito }\end{array}$ & $\begin{array}{l}\text { Abierta al } \\
\text { aprendizaje } \\
\text { colaborativo }\end{array}$ \\
\hline $\begin{array}{l}\text { Sveiby } \\
(1997)\end{array}$ & $\begin{array}{l}\text { Procesos } \\
\text { humanos }\end{array}$ & $\begin{array}{l}\text { Sistemas de } \\
\text { información, } \\
\text { páginas web, } \\
\text { internet, bases } \\
\text { de datos }\end{array}$ & Empresarial & $\begin{array}{l}\text { Miembros de } \\
\text { la } \\
\text { organización } \\
\text { Clientes } \\
\text { Proveedores }\end{array}$ & Formal & Participativo \\
\hline $\begin{array}{l}\text { Bustelo y } \\
\text { Amarilla } \\
(2001)\end{array}$ & $\begin{array}{l}\text { Procesos } \\
\text { humanos } \\
\text { TIC }\end{array}$ & $\begin{array}{l}\text { Bases de datos } \\
\text { corporativas } \\
\text { Aplicaciones } \\
\text { informáticas }\end{array}$ & Empresarial & $\begin{array}{l}\text { Miembros de } \\
\text { la } \\
\text { organización } \\
\text { Expertos en } \\
\text { informática }\end{array}$ & Formal & Participativo \\
\hline
\end{tabular}


Tabla 2

Continuación...

\begin{tabular}{|c|c|c|c|c|c|c|}
\hline Modelo & $\begin{array}{l}\text { Motor de la } \\
\text { GC }\end{array}$ & Uso de TIC & Contexto & Actores & $\begin{array}{c}\text { Tipo de } \\
\text { conocimiento }\end{array}$ & $\begin{array}{c}\text { Cultura } \\
\text { organizacional }\end{array}$ \\
\hline $\begin{array}{l}\text { Kerschberg } \\
(2001)\end{array}$ & TIC & $\begin{array}{l}\text { Páginas web } \\
\text { Correos -e } \\
\text { Bases de datos } \\
\text { Portal } \\
\text { corporativo } \\
\text { Dominios } \\
\text { Mensajería } \\
\text { electrónica } \\
\text { Videos } \\
\text { conferencias } \\
\text { Data mining }\end{array}$ & Empresarial & $\begin{array}{l}\text { Ingenieros de } \\
\text { conocimiento } \\
\text { Expertos } \\
\text { Usuarios de } \\
\text { las TIC } \\
\text { Grupos de } \\
\text { discusión }\end{array}$ & $\begin{array}{l}\text { Tácito } \\
\text { Explícito }\end{array}$ & $\begin{array}{l}\text { Comunicativo } \\
\text { Colaborativo }\end{array}$ \\
\hline $\begin{array}{l}\text { Riesco } \\
(2004)\end{array}$ & $\begin{array}{l}\text { TIC } \\
\text { Procesos } \\
\text { humanos }\end{array}$ & Redes & Empresarial & $\begin{array}{l}\text { Comunidades } \\
\text { de práctica } \\
\text { Equipos de } \\
\text { gestión de } \\
\text { conocimiento }\end{array}$ & $\begin{array}{l}\text { Formal } \\
\text { Experiencias }\end{array}$ & Colaborativo \\
\hline $\begin{array}{l}\text { Paniagua y } \\
\text { López } \\
(2007)\end{array}$ & $\begin{array}{l}\text { TIC } \\
\text { Procesos } \\
\text { humanos }\end{array}$ & $\begin{array}{l}\text { Apoyo de las } \\
\text { TIC (entornos } \\
\text { colaborativos } \\
\text { o entornos de } \\
\text { acceso y } \\
\text { transferencia } \\
\text { del } \\
\text { conocimiento) }\end{array}$ & Empresarial & $\begin{array}{l}\text { Miembros de } \\
\text { la } \\
\text { organización } \\
\text { Expertos } \\
\text { Líder de la } \\
\text { organización }\end{array}$ & $\begin{array}{l}\text { Tácito } \\
\text { Explícito }\end{array}$ & Colaborativo \\
\hline
\end{tabular}

Villasana Arreguín, L. M., Hernández García, P., y Ramírez Flores, E. 
Tabla 2

Continuación...

\begin{tabular}{|l|l|l|l|l|l|l|}
\hline \multicolumn{1}{|c|}{ Modelo } & $\begin{array}{l}\text { Motor de la } \\
\text { GC }\end{array}$ & Uso de TIC & \multicolumn{1}{|c|}{ Contexto } & \multicolumn{1}{|c|}{ Actores } & \multicolumn{1}{c|}{$\begin{array}{c}\text { Tipo de } \\
\text { conocimiento }\end{array}$} & $\begin{array}{c}\text { Cultura } \\
\text { organizacional }\end{array}$ \\
\hline $\begin{array}{l}\text { Angulo y } \\
\text { Negron } \\
(2008)\end{array}$ & $\begin{array}{l}\text { TIC } \\
\text { Procesos } \\
\text { humanos }\end{array}$ & $\begin{array}{l}\text { Páginas web } \\
\text { Software libre } \\
\text { Internet }\end{array}$ & Académico & $\begin{array}{l}\text { Individuos } \\
\text { Grupos }\end{array}$ & $\begin{array}{l}\text { Tácito } \\
\text { Explícito }\end{array}$ & $\begin{array}{l}\text { Comunicativo } \\
\text { Colaborativo }\end{array}$ \\
\hline
\end{tabular}

Fuente: Elaboración propia a partir de Avendaño, V. y Flores, M. (2016).

Otro punto de vista relacionado con la clasificación de los modelos de gestión del conocimiento es el planteado por Rubier (2019) en la tabla 3, quien escribió que es importante comprender el tipo de conocimiento que cada organización puede manejar; a través de este diagnóstico, la organización debe ser capaz de gestionar el conocimiento, y esto tendrá un impacto beneficioso en la organización.

\section{Tabla 3}

Clasificación de los modelos de gestión del conocimiento

\begin{tabular}{|l|l|}
\hline \multicolumn{1}{|c|}{ Autor } & Clasificación \\
\hline McAdam y & $\begin{array}{l}\text { Modelo de clasificación del conocimiento: en este grupo se incluyen algunos } \\
\text { modelos, cuya característica principal es divulgar la gestión del conocimiento } \\
\text { desde una perspectiva conceptual y teórica. }\end{array}$ \\
\cline { 2 - 3 } & $\begin{array}{l}\text { Modelo de capital intelectual: este tipo de modelo asume cómo dividir el capital } \\
\text { intelectual en elementos humanos, de clientes, de procesos y de desarrollo, estos } \\
\text { elementos se encuentran contenidos en dos categorías principales: capital } \\
\text { humano, y estructural / organizacional. }\end{array}$ \\
\cline { 2 - 3 } & $\begin{array}{l}\text { Modelos de conocimiento construidos socialmente: asumen una definición y una } \\
\text { perspectiva más amplias del conocimiento. Estos modelos están intrínsecamente } \\
\text { relacionados con los procesos sociales y el aprendizaje organizacional. }\end{array}$ \\
\hline
\end{tabular}


Tabla 3

Continuación...

\begin{tabular}{|l|l|}
\hline \multicolumn{1}{|c|}{ Autor } & Clasificación \\
\hline $\begin{array}{l}\text { Rodríguez Gómez } \\
\text { (2006) }\end{array}$ & $\begin{array}{l}\text { Almacenamiento, acceso y transferencia de conocimiento: estos modelos se } \\
\text { enfocan en la creación de métodos, estrategias y tecnologías que puedan } \\
\text { almacenar conocimiento y facilitar el acceso y posterior transferencia de } \\
\text { conocimiento entre los miembros de la organización. }\end{array}$ \\
\cline { 2 - 3 } & $\begin{array}{l}\text { Sociocultural: se basan en promover el desarrollo de la cultura organizacional, } \\
\text { que promueve la generación de procesos de gestión del conocimiento. }\end{array}$ \\
\cline { 2 - 3 } & $\begin{array}{l}\text { Tecnológicos: este tipo de modelos se enfocan en el desarrollo y uso de sistemas } \\
\text { informáticos y herramientas técnicas para la gestión del conocimiento. }\end{array}$ \\
\hline \multirow{5}{*}{$\begin{array}{l}\text { Kakabadse, } \\
\text { Kouzmin (2003) }\end{array}$} & $\begin{array}{l}\text { Modelos filosóficos de gestión del conocimiento: estos modelos están } \\
\text { relacionados con la epistemología o la propia composición del conocimiento. } \\
\text { Estos intentan explicar cómo obtener información de la realidad social y } \\
\text { organizacional. }\end{array}$ \\
\cline { 2 - 3 } & $\begin{array}{l}\text { Modelos cognitivos de gestión del conocimiento: están relacionados con la } \\
\text { ciencia positivista y representan mecanismos para comprender la causalidad. }\end{array}$ \\
\cline { 2 - 3 } & $\begin{array}{l}\text { Modelo de red de gestión del conocimiento: este tipo de modelo surge junto con } \\
\text { la teoría de la organización en red y se enfoca en la adquisición, intercambio y } \\
\text { transferencia de conocimiento, este conocimiento es el aspecto básico del } \\
\text { aprendizaje organizacional, permitiendo la selección y adopción de nuevas } \\
\text { prácticas cuando se considere relevante. }\end{array}$ \\
\cline { 2 - 3 } & $\begin{array}{l}\text { Modelo de comunidad de práctica de gestión del conocimiento: su fundamento se } \\
\text { construye desde la perspectiva de la sociología y la historia. }\end{array}$ \\
\hline
\end{tabular}

Villasana Arreguín, L. M., Hernández García, P., y Ramírez Flores, E. 


\section{Tabla 3}

Continuación...

\begin{tabular}{|l|l|}
\hline \multicolumn{1}{|c|}{ Autor } & Clasificación \\
\hline $\begin{array}{l}\text { Kakabadse, } \\
\text { Kakabadse y }\end{array}$ & $\begin{array}{l}\text { El modelo cuántico de gestión del conocimiento: su fundamento se basa en un } \\
\text { punto de vista cuántico, que se basa en trabajos en física cuántica, tecnologías } \\
\text { cuánticas emergentes y economía. }\end{array}$ \\
\hline Barragán (2009) & $\begin{array}{l}\text { Conceptos, teorías y filosofía: la principal característica es enriquecer el modelo } \\
\text { de investigación en gestión del conocimiento a través de la epistemología y las } \\
\text { teorías y métodos conceptuales de temas relacionados con el conocimiento. }\end{array}$ \\
\cline { 2 - 3 } & $\begin{array}{l}\text { Cognoscitivos y de capital intelectual: generalmente se desarrolla dentro de } \\
\text { organizaciones e industrias que buscan enfocarse en el uso y aplicación del } \\
\text { conocimiento para crear valor para sus productos y procesos. }\end{array}$ \\
\cline { 2 - 3 } & $\begin{array}{l}\text { Sociales y de trabajo: en este campo, la característica principal del modelo de } \\
\text { diferenciación es estudiar la socialización del conocimiento entre diferentes } \\
\text { actores o grupos de trabajo para comprender y optimizar el mecanismo de uso y } \\
\text { transferencia del conocimiento para promover intereses sociales y / o grupales. }\end{array}$ \\
\cline { 2 - 3 } & $\begin{array}{l}\text { Técnicos y científicos: los modelos técnicos y científicos forman parte de esta } \\
\text { categoría, incluidos los modelos que buscan combinar modelos que utilizan las } \\
\text { TIC para mejorar el uso y la aplicación del conocimiento. }\end{array}$ \\
\cline { 2 - 3 } \\
$\begin{array}{l}\text { Modelos holísticos de gestión del conocimiento: este grupo incluye modelos } \\
\text { cuyas características no son adecuadas para los primeros cuatro grupos descritos, } \\
\text { o cuyo contenido tiene dos o más características de los grupos discutidos } \\
\text { anteriormente, por lo que no se les permite ser clasificados explícitamente en } \\
\text { ninguna categoría propuesta anteriormente. }\end{array}$ \\
\hline
\end{tabular}




\section{Tabla 3}

Continuación...

\begin{tabular}{|l|l|}
\hline \multicolumn{1}{|c|}{ Autor } & Clasificación \\
\hline Marulanda et al. & $\begin{array}{l}\text { Modelos con enfoque desde la concepción: cuando el enfoque conceptual se } \\
\text { centra en el tema, se dividen en modelos temáticos; cuando el enfoque conceptual } \\
\text { se centra en la organización, se dividen en modelos temáticos. }\end{array}$ \\
\cline { 2 - 3 } & $\begin{array}{l}\text { Modelos organizacionales: se categorizan según el tipo de organización, al incluir } \\
\text { esas visiones de la organización, como Taylor Ana y Fioriana, hacen de las } \\
\text { personas un simple engranaje o unidad funcional en el proceso de producción } \\
\text { tecnológica, luego pueden ser mecánicas. Cuando los organismos se inspiran en } \\
\text { los organismos, sus necesidades y la relación de supervivencia con el medio, son } \\
\text { los elementos estructurales de la nueva versión organizacional; cada miembro de } \\
\text { la organización participa de las metas y conocimientos de toda la empresa, y } \\
\text { busca sistemáticamente cambios organizacionales. Cuestiones empresariales } \\
\text { integrales y holísticas. }\end{array}$ \\
\cline { 2 - 2 } & $\begin{array}{l}\text { Modelos desde el tipo de organización: cuando las empresas tradicionales se } \\
\text { consideran generalmente como organizaciones que adquieren conocimientos } \\
\text { mediante la contratación de personal especializado, se clasifican como } \\
\text { organizaciones tradicionales. Orientada hacia la realización de la innovación de la } \\
\text { cultura de la innovación, esta cultura de la innovación puede mejorar o mejorar la } \\
\text { sinergia que tienen las organizaciones y las organizaciones híbridas que combina } \\
\text { los dos primeros conceptos. }\end{array}$ \\
\hline $\begin{array}{l}\text { Modelos de gestión: se dividen en cultura organizacional, gestión del cambio, } \\
\text { gestión estratégica, gestión según capacidad, gestión según procesos y gestión de } \\
\text { la información. }\end{array}$ \\
\hline
\end{tabular}

Fuente: Elaboración propia a partir de Rubier, D. (2019).

Villasana Arreguín, L. M., Hernández García, P., y Ramírez Flores, E.
Vol. 6, núm. 18 / septiembre - diciembre del 2021 DOI: https://doi.org/10.36791/tcg.v0i18.128

Pp. 53-78 


\section{Estado actual de la gestión del conocimiento}

Se puede decir que la gestión del conocimiento no solo es actual, sino que existe en la literatura desde hace mucho tiempo. El análisis más convincente es el análisis bibliométrico, que simplemente cuenta el número de artículos en la literatura empresarial y lo compara con otras pasiones empresariales. La mayor parte del entusiasmo empresarial creció rápidamente y alcanzó su punto máximo después de unos cinco años, y luego disminuyó casi tan rápido como el crecimiento (Koenig, 2018). En la figura 1, puede ver la evolución conceptual.

\section{Figura 1}

Gráfico de tendencia de las citas del concepto gestión del conocimiento de 2001 a 2011

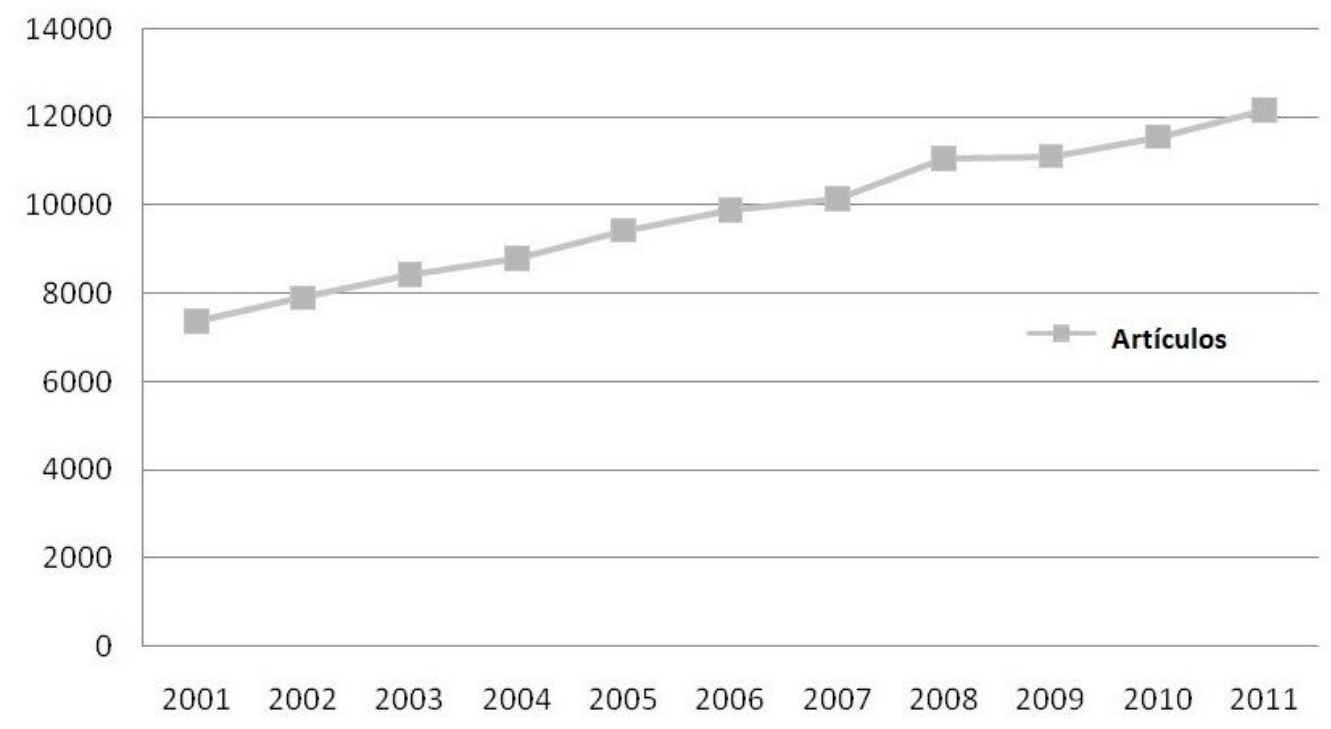

Fuente: Elaboración propia a partir de Koenig, M. (2011). 
Desde esta perspectiva, la tasa de éxito de la gestión del conocimiento en una organización se relaciona con la posibilidad de utilizar diferentes conocimientos para adquirir nuevas fases, que pueden mejorar las acciones organizacionales o sentar las bases para nuevas acciones. Cómo promueven el desempeño de la innovación y guían el sistema de conocimiento de la organización para generar nuevas ideas, mejorando así las capacidades de innovación (Rubier, 2019).

Girard y Ribière (2016) escriben que, respecto al futuro de la gestión del conocimiento, dado que este ha sido el campo de mayor interés para académicos y profesionales desde su creación, converge en el hecho de que las organizaciones confían cada vez más en el conocimiento para operar y competir (economía del conocimiento). Por tanto, el foco de la controversia sigue siendo si mantendrá el mismo nombre "gestión del conocimiento" y seguirá siendo responsable del "departamento de gestión del conocimiento", o si se convirtiese en una corriente principal y pasará a formar parte de todas las actividades en los diferentes niveles y disciplinas de la organización.

Respecto al futuro de la gestión del conocimiento, dado que este ha sido el campo de mayor interés para académicos y profesionales desde su creación, converge en el hecho de que las organizaciones confían cada vez más en el conocimiento para operar y competir (economía del conocimiento). Por tanto, es inevitable La tierra debe ser gestionada de forma adecuada y sistemática. El foco de la controversia sigue siendo si mantendrá el mismo nombre "gestión del conocimiento" y seguirá siendo responsabilidad del "departamento de gestión del conocimiento", o si se convertirá en una corriente principal y pasará a formar parte de todas las actividades en los diferentes niveles y disciplinas de la organización (Girard y Ribière, 2016).

Casado (2020) enfatizó que el capital del conocimiento es cada vez más reconocido como el valor diferenciado de las empresas. En las últimas décadas, el desarrollo del concepto ha sido paralelo a la tecnología que lo sustenta. Inicialmente, esta tecnología solo se utilizaba como soporte para el procesamiento de datos. Se ha convertido en sistemas de información cada vez más complejos y tiene funciones más completas. Ahora las nuevas tecnologías digitales nos permiten analizar y predecir lo que sucederá, y hacerlo, y tomar una decisión sobre el pasado.

En la búsqueda de conocimientos aplicados, ideas y tecnologías innovadoras, las empresas son cada vez más conscientes de la importancia de mantenerse en contacto con clientes, socios, proveedores, inventores aficionados, investigadores académicos, científicos, intermediarios de innovación y otros agentes. Expresiones y términos como crear comunidad, red de innovación, innovación de mercado abierto u originalidad de los fans (crowdsourcing) los cuales se utilizan para generar conocimiento y hablar sobre el futuro de la innovación y el trabajo en un mundo globalizado. Por lo tanto, con la ayuda del capital intelectual externo, muchos gerentes tienen la máxima prioridad para expandir su visión del conocimiento y la innovación organizacional. El nuevo paradigma tiene que ver con la actitud de compartir conocimientos de manera generosa y desinteresada. Pero en esta nueva situación, compartir significa incluso renunciar a bienes y derechos de propiedad intelectual, porque ahora la gestión del conocimiento se basa en compartir, más que proteger el conocimiento en sí, porque es fundamental y más allá de la tradición, muestra el desarrollo de la gestión del conocimiento (ver figura 2).
Villasana Arreguín, L. M., Hernández García, P., y Ramírez Flores, E. 


\section{Figura 2}

Evolución de la gestión del conocimiento

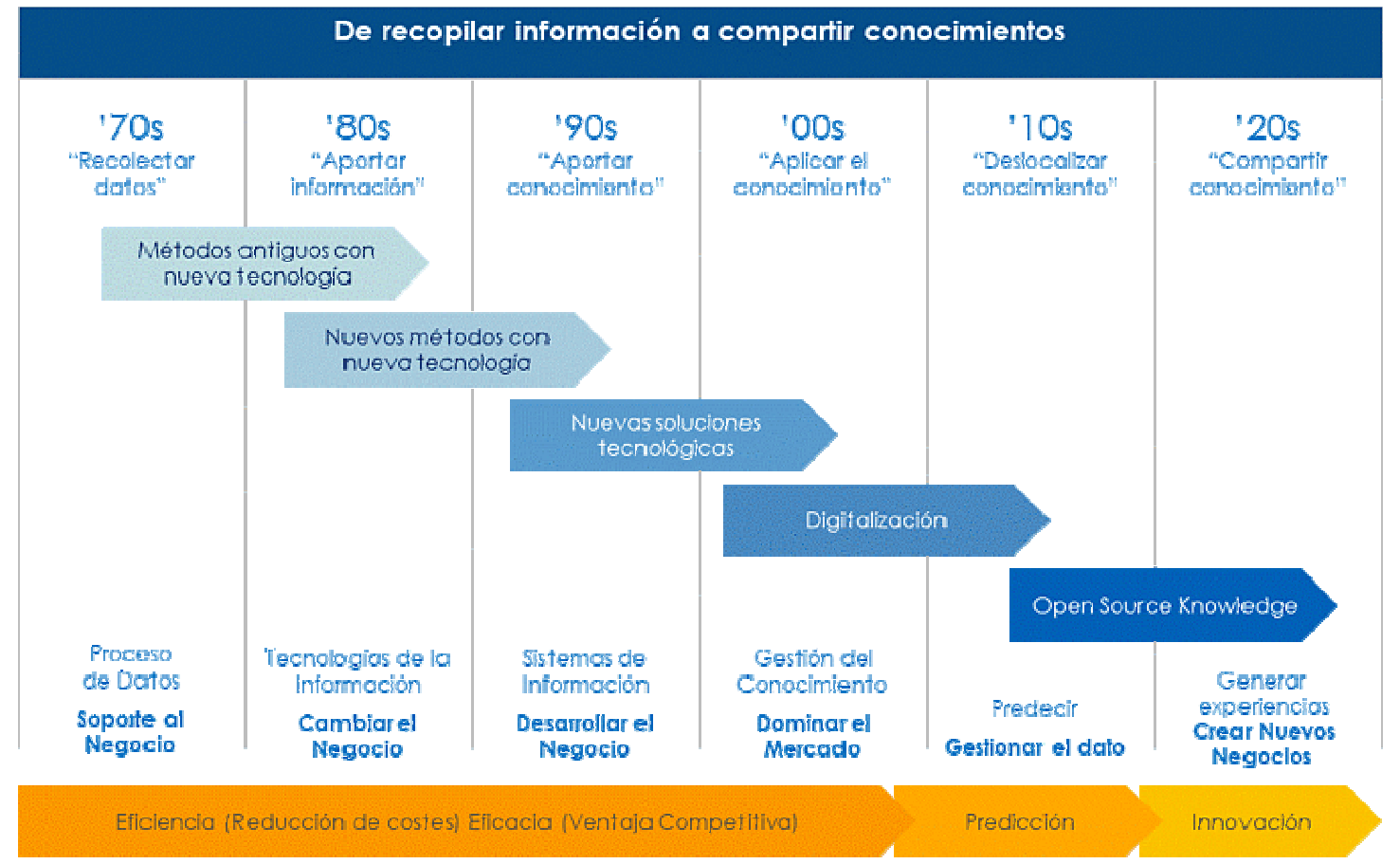

Fuente: Casado, J. M. (2020).

Otro desafío de futuro es vincular la gestión del conocimiento con la Industria 4.0. El término Industria 4.0 hace referencia a la llamada cuarta revolución industrial impulsada por la transformación digital, que significa la organización y gestión de la cadena de valor del sector, en donde se produjo un salto cualitativo. (Buisán y Valdés, 2017). Por su parte, Blanco, Fontrodona y Poveda (2017) explicaron las nueve tecnologías que están cambiando la producción industrial, como se muestra en la figura 3, las cuales son big data y análisis, robots autónomos, simulación, integración horizontal y vertical de sistemas, internet industrial de las cosas, redes seguridad, nube, fabricación aditiva y realidad aumentada:

- Big data y análisis: incluye el análisis de conjuntos de datos, que están más allá de las capacidades de los sistemas informáticos ordinarios debido a su número, naturaleza y velocidad que deben procesarse. En el contexto de la Industria 4.0, el análisis de big data (sistemas y equipos de producción, sistemas de gestión de proveedores, etc.) se convertirá en el estándar para apoyar la toma de decisiones en tiempo real. 
- Robots autónomos: los robots se están volviendo cada vez más autónomos, flexibles y colaborativos para que puedan interactuar entre sí y trabajar juntos de forma segura y aprender de los humanos. Estos robots serán más económicos: Sirkin y otros. dar. El informe de investigación de Boston Consulting Group (2015) predice que el precio de los robots y el software caerá un $20 \%$ en los próximos diez años. En comparación con la situación actual, también tienen mayores posibilidades (se espera que aumenten los ingresos en un 5\% anual). Esto conducirá a más tareas en las que es rentable reemplazar la mano de obra con robots, en donde la tasa de crecimiento anual del número de robots aumentará del 2-3\% actual al 10\% en los próximos diez años.

- Simulación: la simulación 3D que se encuentra actualmente ampliada en la fase de ingeniería también se utilizará en algunas operaciones de la planta de producción. Permitirán reproducir el mundo físico en un modelo virtual, que puede contener máquinas, productos y personas, y permitirán a los operadores probar y optimizar la programación de la máquina en el mundo virtual antes de ponerla en funcionamiento.

- Integración horizontal y vertical del sistema: fabricantes, proveedores y clientes estarán estrechamente vinculados a través de sistemas informáticos para promover una cadena de valor verdaderamente automatizada. Lo mismo puede ocurrir entre varios departamentos de la empresa, como los departamentos de ingeniería, producción y servicio.
- Internet industrial de las cosas (IoT): cada vez más dispositivos estarán equipados con funciones informáticas integradas y conectados a través de tecnologías estándar. Esto permite que los dispositivos de campo se comuniquen e interactúen entre sí y con el controlador central. También descentraliza el análisis y la toma de decisiones y realiza una respuesta en tiempo real.

- Seguridad cibernética: la conectividad mejorada que representa la Industria 4.0 ha aumentado enormemente la necesidad de proteger los sistemas industriales críticos y las líneas de producción de las amenazas cibernéticas. También debemos mejorar la protección de la propiedad intelectual, los datos personales y la privacidad.

- Nube: cada vez más tareas relacionadas con la producción requerirán más intercambio de datos. $\mathrm{Al}$ mismo tiempo, la tecnología en la nube mejorará y logrará tiempos de respuesta de solo unos pocos milisegundos. Como resultado, el trabajo de TI se transferirá a la nube, lo que facilitará la puesta en producción de más servicios de TI. Incluso el sistema que controla el proceso puede estar basado en la nube.

- Fabricación aditiva: la impresión 3D, además de la producción actual de prototipos y componentes individuales, también se ampliará para producir pequeños lotes de productos personalizados, lo que reducirá las distancias de materias primas, inventario y transporte.

Realidad aumentada: los operadores equipados con gafas de realidad aumentada pueden, por ejemplo, recibir instrucciones sobre cómo reparar la máquina en
Villasana Arreguín, L. M., Hernández García, P., y Ramírez Flores, E.
Vol. 6, núm. 18 / septiembre - diciembre del 2021 DOI: https://doi.org/10.36791/tcg.v0i18.128

Pp. $53-78$ 
el trabajo. También existen aplicaciones en el campo de la formación. En el futuro, la empresa proporcionará a los trabajadores información en tiempo real de forma más amplia para mejorar la toma de decisiones y los procedimientos laborales (figura 3).

\section{Figura 3}

Las nueve tecnologías que están transformando la producción industrial

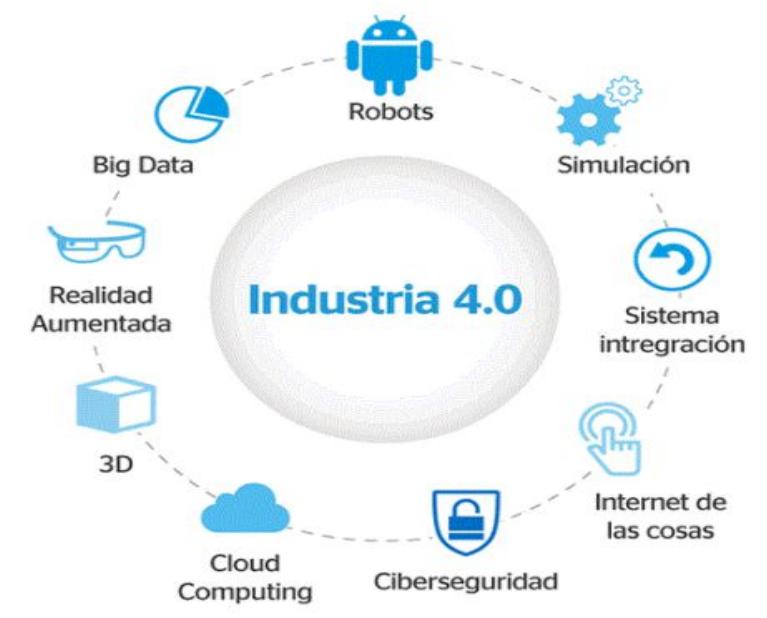

Fuente: Blanco, R., Fontodrona, J. y Poveda, C. (2017).

\section{Metodología}

Para lograr las metas propuestas en este trabajo, se realizó un análisis de acuerdo con el proceso propuesto por Levy y Ellis (2006). El proceso sugirió: 1) revisar los ítems de calidad de los artículos recomendados, es decir, se clasifican como de alta - las bases de datos de calidad desde el exterior 2) el proceso de análisis, síntesis y categorización de estos elementos de acuerdo con el propósito de este artículo; 3) la salida de los resultados de la disputa (ver figura 4). Hart (1998) define la crítica literaria como utilizar las ideas de la literatura para probar el método específico del tema, la elección del método y la investigación que contribuya a la demostración de algunas cosas nuevas. Señaló que, en la literatura, calidad significa amplitud y profundidad adecuadas, rigurosas y consistentes, claras y breves, y análisis y síntesis efectivos. J. Shaw (1995, citado por Levy y Ellis, 2006) señaló que el proceso de revisión debería explicar cómo un estudio se basa en otro. Webster y Watson (2002, citado por Levy y Ellis, 2006). La revisión efectiva de la literatura se define como sentar una base sólida para el desarrollo del conocimiento (figura 4). Promueve el desarrollo de la teoría, cierra los campos que requieren mucha investigación y descubre los campos que necesitan investigación. De acuerdo con estas definiciones, es obvio que una revisión de la literatura efectiva debe incluir las siguientes características: a) análisis metodológico y 
síntesis de literatura de alta calidad, b) proporcionar una base sólida para los temas de investigación, y c) proporcionar una base sólida para la investigación. elección de los métodos de investigación, d) demostrar que la investigación propuesta ha hecho nuevas contribuciones al sistema de conocimiento o promovido la base de conocimiento de Levy y Ellis (2006) en el campo de la investigación.

\section{Figura 4}

Las tres etapas del proceso de revisión efectiva de la literatura

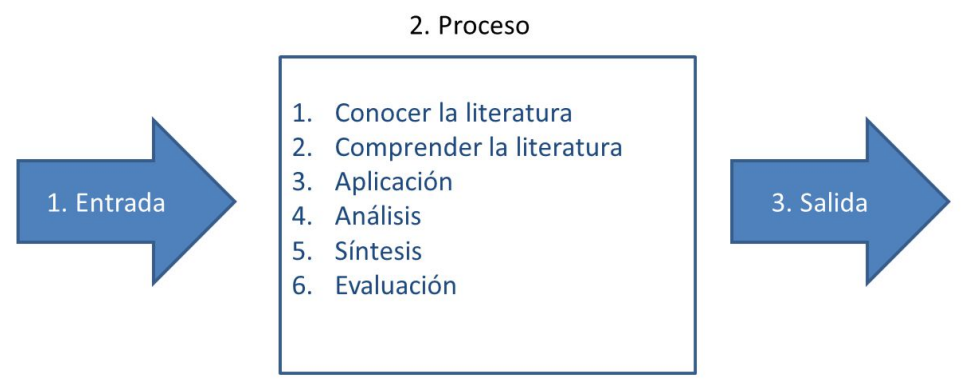

Fuente: Levy, Y. y Ellis, T. (2006).

\section{Resultados}

Los resultados de la investigación bibliográfica respondieron a las preguntas planteadas en la investigación y dieron respuesta a las preguntas sobre la definición de conocimiento, los resultados mostraron que se encontraron diferentes definiciones del concepto de gestión del conocimiento, que se concentran en la tabla 1. En la literatura se puede observar que el concepto continúa desarrollándose en el tiempo, pero esta definición corta y precisa representa el significado de la gestión del conocimiento de manera general: la gestión del conocimiento es el proceso de captura,
Villasana Arreguín, L. M., Hernández García, P., y Ramírez Flores, E. distribución y uso efectivo del conocimiento (Davenport, 1994). Para responder preguntas sobre las teorías que sustentan la gestión del conocimiento, las personas descubrieron la teoría de los recursos y la capacidad de Penrose (1959); Barney (1991); y la teoría de la creación de conocimiento organizacional propuesta por Nonaka y Takeuchi (1995). Por otro lado, para responder a la existencia de modelos de gestión del conocimiento, se encontraron diferentes modelos utilizados en la gestión del conocimiento, entre los que destacan los siguientes (Romero, 2009):
Vol. 6, núm. 18 / septiembre - diciembre del 2021 DOI: https://doi.org/10.36791/tcg.v0i18.128

Pp. $53-78$ 
- Modelo en espiral de conocimiento o proceso de creación de conocimiento (Nonaka y Takeuchi, 1995). -El modelo se basa en el proceso de interacción entre dos tipos de conocimiento tácito y conocimiento explícito, el cual se caracteriza por su domesticación dinámica y sigue en cuatro etapas: socialización, externalización, integración e internalización; este Movimiento produce una espiral de conocimiento.

- Herramienta de evaluación de la gestión del conocimiento (KMAT). -Herramienta de evaluación y diagnóstico basada en el modelo de gestión del conocimiento organizacional desarrollado por Arthur Andersen y APQC. KMAT plantea cuatro factores (liderazgo, cultura, tecnología y medición), que son propicios para el proceso de gestión del conocimiento organizacional.

- El modelo de Arthur Andersen - refuerza la importancia de que los empleados compartan y aclaren los conocimientos, y plantea la necesidad de acelerar el flujo de información valiosa de las personas a las organizaciones y a las personas para que puedan usarla para crear valor para los clientes. Su novedad es que, desde una perspectiva individual, los individuos tienen la responsabilidad de compartir conocimientos para la organización y dejarlo claro; desde una perspectiva organizacional, también significa la responsabilidad de establecer infraestructura de apoyo, por lo que el punto de vista del individuo es efectivo Desarrollar procesos, culturas, tecnologías y sistemas que permitan la adquisición, análisis, síntesis, aplicación, evaluación y distribución de conocimientos.
- Modelo de gestión del conocimiento de consultoría de KPMG - revelar los factores que restringen la capacidad de aprendizaje de la organización y los resultados esperados. Una de las características básicas del modelo es la interacción de todos sus elementos, que parece ser un sistema complejo en el que los efectos ocurren en todas direcciones. La estructura organizativa, la cultura, la capacidad de liderazgo, el mecanismo de aprendizaje, la actitud de las personas, la capacidad de trabajo en equipo, etc., no son independientes, sino que están interconectados.

Para dar respuesta a preguntas sobre aplicaciones de gestión del conocimiento, se encontró que se aplica a través de un modelo, que enfatiza que las organizaciones necesitan sistematizar y estructurar los procesos relacionados con el conocimiento para obtener diferentes beneficios. Para responder preguntas sobre los beneficios de la gestión del conocimiento, algunos de los beneficios encontrados son:

- Incrementar la creatividad

- Genera nuevas ideas

- Mejora de la capacidad de innovación

- Ayudar a la empresa a ser más rápida, más eficiente o innovadora que sus competidores

- Enfrentar desafíos ambientales y mantener la competitividad del mercado

- Mejorar el rendimiento, con un enfoque en mejorar la eficiencia, la eficacia y la innovación

- Lograr metas y optimizar el proceso de toma de decisiones 
- Adquirir capacidad competitiva para asegurar su capacidad de supervivencia y supervivencia

- Obtenga el mayor valor del conocimiento existente

- Crear una ventaja competitiva

- Promover las habilidades organizativas y la creación de valor

- Identificar y utilizar activos intangibles existentes y generar nuevos activos intangibles

- Buen funcionamiento de los procesos en la organización

- Contribuir a la toma de decisiones para mejorar la viabilidad y durabilidad de la organización y generar una ventaja competitiva

- Proporcionar valor a la organización para lograr habilidades o habilidades básicas únicas

- Incrementar la producción de conocimiento organizacional

- Mejorar la comprensión de las personas sobre áreas específicas de interés

Para dar respuesta a las expectativas de la futura gestión del conocimiento, se puede demostrar que las organizaciones se apoyan cada vez más en el conocimiento para operar y competir (economía del conocimiento), por lo que es inevitable realizar una gestión adecuada y sistemática. El nuevo paradigma tiene que ver con la actitud de compartir conocimientos de manera generosa y desinteresada. Pero en esta nueva situación, compartir significa incluso renunciar a activos y derechos de propiedad intelectual, porque ahora la gestión del conocimiento, en lugar de proteger el conocimiento en sí, se basa en el intercambio de conocimientos, porque es fundamental y está más allá de la tradición.

Otro desafío de futuro es vincular la gestión de la gestión del conocimiento con la Industria 4.0. (Blanco, Fontodrona y Poveda, 2017) explicaron las nueve tecnologías que están cambiando la producción industrial, como se muestra en la figura 3 , tales como big data y análisis, autónomos, robótica, simulación, integración horizontal y vertical de sistemas, Internet industrial de las cosas, ciberseguridad, nube, fabricación aditiva y realidad aumentada.

\section{Conclusiones}

En la revisión de la literatura, se muestra que la gestión del conocimiento ha hecho un gran aporte a la creación de ventajas competitivas en las organizaciones basadas en el conocimiento. La creación, intercambio, combinación y aplicación de información no solo se relaciona con la enseñanza y el aprendizaje, sino que también se gestiona la organización. a través de sistemas de gestión Operar mejor y responder a las necesidades internas y externas, mejorando así la eficiencia de los procesos y servicios.

La importancia de la gestión del conocimiento destaca que la globalización es un desafío para probar el conocimiento organizacional. El uso de herramientas técnicas es fundamental para el funcionamiento normal de una organización, pero el desafío principal sigue siendo cómo transformar los talentos en el patrimonio de conocimiento de la organización. Con el fin de optimizar el proceso de toma de decisiones, operación, ejecución de la estrategia y realización de objetivos, de
Villasana Arreguín, L. M., Hernández García, P., y Ramírez Flores, E. 
modo que el conocimiento en la mente de las personas pueda transformarse de implícito a explícito y hacerlo aplicable.

El modelo teórico que se presenta en este documento se adapta a las diferentes situaciones y culturas de la organización, es una opción para visualizar la gestión del conocimiento de forma sistemática y estructurada, todo depende de la identidad de la empresa y de sus capacidades. Poner en práctica. La gestión del conocimiento fomenta diversos métodos de combinación horizontal, como el trabajo en equipo, multidisciplinario, interdisciplinario e interdisciplinario, y utilizarlos como recursos para la creación de nuevos conocimientos y tecnologías.

\section{Referencias}

Abellón, O., y Rojo, T. (2004). Brainworkers: Trabajadores del conocimiento. Aposta Revista de Ciencias Sociales, 5, 1-5.

Abeson, F., y Taku, M. A. (2009). Knowledge source and small business competitiveness. Competitiveness Review: An International Business Journal incorporating Journal of Global Competitiveness, 19(2), 88-95.

https://doi.org/10.1108/10595420910942252

Alavi, M., y Leidner, D. (2001). Review: Knowledge management and knowledge management systems: conceptual foundations and research issues. MIS Quarterly, 25(1), 107-136.

https://doi.org/10.2307/3250961

Avendaño, V., y Flores, M. (2016). Modelos teóricos de gestión del conocimiento: descriptores, conceptualizaciones y enfoques. Entre ciencias: Diálogos en la Sociedad del Conocimiento, 4(10), 201-227. https://doi.org/10.21933/j.edsc.2016.10.181
Barney, J. (1991). Firm Resources and Sustained Competitive Advantage. Journal of Management, 17(1), 99-120.

Bellinza, M., Guerrero, N., Colon, S., y Ramírez, W. (2011). Gestión del conocimiento: Aproximaciones teóricas. CLIO América, 5(10), 257-271. https://doi.org/10.21676/23897848.422

Bueno, E. (1998), Modelo Intelect, Euroforum Escorial, San Lorenzo del Escorial.

Bueno, E. (1998). El capital intangible como clave estratégica en la competencia actual. Boletín de Estudios Económicos 53, 207-229.

Blanco, R., Fontodrona, J., y Poveda, C. (2017). La industria 4.0: El estado de la cuestión. Economía Industrial, 406, 151-164.

Borroto, M. J. (2007). La Gestión Del Conocimiento En La Nueva Economía. Algunos Apuntes. Ciencia En Su PC, 5, 30-40.

Buisán, M., y Valdés, F. (2017). La industria Conectada 4.0. Información Comercial Española, ICE: Revista de Economía, 898, 89-100.

https://doi.org/10.32796/ice.2017.898.1963

Casado, J. M. (2020). Más allá de la gestión tradicional del conocimiento. 2.C Consulting. https://www.dosc.es/2020/01/30/mas-alla-de-lagestion-tradicional-del-conocimiento/

Chatti, M. A. (2012). Knowledge management: A personal knowledge network perspective. Journal of Knowledge Management, 16(5), 829-844. https://doi.org/10.1108/13673271211262835

Davenport, T.H. (1994): The Coming Soon: The CKO, InformationWeek, September 5.
Villasana Arreguín, L. M., Hernández García, P., y Ramírez Flores, E. 
Davenport, T. H., y Prusak, L. (1998). Working Knowledge How Organization Manage What They Know. (Harvard Business School Press, Ed.) (1-199). Boston, Massachusetts, United States of America.

Dabić, M., Lažnjak, J., Smallbone, D., y Švarc, J. (2019). Intellectual capital, organisational climate, innovation culture, and SME performance: Evidence from Croatia. Journal of Small Business and Enterprise Development, 26(4), 522-544. https://doi.org/10.1108/JSBED-04-2018-0117

Edmonson, R. R. (2010). Knowledge management practices within Hong Kong organizations. Journal of Knowledge-based Innovation in China, 2(2), 213-232. https://doi.org/10.1108/17561411011054805

Girard, J., y Ribière, V. (2016). Mapping the future of KM through Earl's KM taxonomy lens. Online Journal of Applied Knowledge Management, 4(1), 180-191. https://doi.org/10.36965/ojakm.2016.4(1)180-191

Greiner, M. E., Böhmann, T., y Krcmar, H. (2007). A strategy for knowledge management. Journal of Knowledge Management, 11(6), 3-15. doi:10.1108/13673270710832127

Holtshouse, D. (2010). Knowledge work 2020: thinking ahead about knowledge work. On the Horizon, 18(3), 193-203. doi:10.1108/10748121011072645

Kristandl, G., y Bontis, N. (2007). Constructing a definition for intangibles using the resource-based view of the firm. Management Decision, 45(9), 1510-1524. https://doi.org/10.1108/00251740710828744

Koenig, M. (2018), What is KM? Knowledge Management Explained. KM World https://www.kmworld.com/About/What_is_Knowledge _Management
Levy, Y., y Ellis, T. J. (2006). A Systems Approach to Conduct an Effective Literature Review in Support of Information Systems Research. Informing Science Journal, 9(1), 181-212. https://doi.org/10.28945/479

Ngah, R., y Ibrahim, A. R. (2010). The influence of intellectual capital on knowledge sharing: Small and Medium Enterprises perspective. Business Transformation through Innovation and Knowledge Management: An Academic Perspective - Proceedings of the 14th International Business Information Management Association Conference, IBIMA 2010, 2, 638-654.

https://www.scopus.com/inward/record.uri?eid=2-s2.084905089851\&partnerID $=40 \&$ md5 $=$ d3024b31be4853b 16e3175694eaf1b99

Nonaka, I., y Takeuchi, H. (1995). The Knowledge Creating Company. (O. USA, Ed.) (284). New York, NY, United States of America: Oxford University Press.

Penrose, E. T. (1959). The theory of the growth of the firm. New York: John Wiley. Pisano

Reed, K., Lubatkin, M., y Srinivasan, N. (2006), Proposing and testing an intellectual capital-based view of the firm, Journal of Management Studies, 43(4), 867-893. https://doi.org/10.1111/j.14676486.2006.00614.x

Romero, F. T. (2016). Información de los recursos intangibles ocultos: ¿Memorias de sostenibilidad o informe anual? European Research on Management and Business Economics, 22(2), 101-109. https://doi.org/10.1016/j.iedee.2015.06.001
Villasana Arreguín, L. M., Hernández García, P., y Ramírez Flores, E.
Vol. 6, núm. 18 / septiembre - diciembre del 2021 DOI: https://doi.org/10.36791/tcg.v0i18.128

Pp. 53-78 
Rubier, D. (2019). La incidencia de la gestión del conocimiento en el éxito de las organizaciones. Cooperativismo y Desarrollo, 7(3), 392-405.

http://coodes.upr.edu.cu/index.php/coodes/article/view/2 69

Secundo, G., Del Vecchio, P., Dumay, J., y Passiante, G. (2017). Intellectual capital in the age of Big Data: establishing a research agenda. Journal of Intellectual Capital, 18(2), 242-261. https://doi.org/10.1108/JIC-102016-0097

Subramaniam, M., y Youndt, M. A. (2005). The Influence of Intellectual Capital on the Types of Innovative Capabilities. Management, 48(3), 450-463.

https://doi.org/10.2307/20159670
Sveiby, K. E. (2018). Measuring Intangibles: Suggested indicators with cases from professional service organizations and high-tech firms. http://www.sveiby.com/articles/

Tenorio, J., Popadiuk, S., y Santos, N. (2020). Knowledge management at Unilever South America - enabling conditions and their interaction with the SECI model. Revista de Negocios Studies on Emerging Countries, 24(2), 7-26.

https://doi.org/10.7867/1980-4431.2019v24n2p7-26 